buried between them, seen in Fig. 3, is a well. marked peculiarity, the one generally represented in Punch. The other symptoms which give the disease its name (acromegaly, big ends) are to be found in the large hands and feet in some of the pictures. The fingers are increased in length but not in breadth (Pierre Marie's type en long) (Fig. 2). In two pictures the feet are large and everted. The knock-knee and the bent-up condition of the lower extremities seen in all the pictures is due to some paralysis. The patient has probably in childhood suffered from rickets. He really seems to suffer from a variety of complaints, including some rheumatic affection of his left hand. The ulnar deflection is well depicted. (Fig. 1.)

The writer is indebted to Mr. Meredith $T$. Whittaker, J.P., of Weaponness Park, Scarborough, for the loan of five pictures and for the trouble he has taken in kindly supplying information about this curious individual. Mr. Whittaker has a very large collection of pictures and prints connected with Scarborough, and being most interested in the fact that possibly the earliest representations of a rare disease, only recognised of late years, are to be found at Scarborough, he has been good enough to write:--

I send you five portraits of Dickinson. I think the etching best answers your purpose. I have the companion to it framed showing Dickie seated on his chest and his head tilted sideways, which displays the enormous length of his jaw. Probably the pictures vary on account of the portrait being drawn at different periods of the disease. I do not know the date of the etching. I have a copp of the one by Virtue ; this was issued in 1725 .

Dickie had a trip to London, and it was probable on that occasion when he was painted.

Settrington, who was a local bookseller, published a portrait of him in 1726, which I send. The two mezzotints are rare and one is in excellent preservation. These appear to me to be copied from Hysing, although the position is altered. Dickie was born in 1670 ; he was appointed as governor of the Spa in 1700, and had charge of the lavatories. He was probably of a low type of mind of an immoral age. According to the pictures his house was one of good class, and I have seen it recorded that it was showily furnished. The landslide of February, 1737-8, would cause his property great damage, and he apparently never got over the shock, and he died some time in 1738. His age would be 68 .

It is interesting to think that we have here, perhaps, the earliest representation of an acromegalic to be found in England. Some earlier ones have been recognised on the continent-for instance, the portrait of a "giant" at the court of the Elector of the Palatinate, Frederick II., preserved in the Ambras-Schloss in the Tyrol, painted life size in 1553 by his court painter.

Another of still earlier date, of special interest at the present moment, is a carved figure of a woman, dating from the thirteenth century, which was placed high up on one of the flying buttresses of Reims Cathedral. This statue has been described as pointing out in a striking way the physical characteristics of acromegaly. As it stood on the north side of the cathedral, which was the one which suffered least from the early bombardment, there were hopes that it had been spared. At the moment of writing, however, the account of a renewed bombardment of Reims leaves one little hope that this interesting relic of the past, after weathering the storms of seven centuries, is not now to be reckoned amongst the innocent victims who have fallen before the spread of German Kultur.

Oxford-terrace, Hyde Park, $W$.

\section{TRIGGER FINGER, ITS CAUSE AND MECHANISM.}

Bx ADONIRAM B. JUDSON, M.D.

A PROFESSIONAL man, 76 years old, had an attack of arthritis in the fingers of both hands in June, 1913, with resulting trigger finger in the proximal phalangeal joint of the middle finger of the right hand, with swelling and deformity, and stiffness of the joints of the middle and ring fingers. These results are still present. Motion past the right angle in either flexion or extension develops a painful snap or jerk. This may be described as a momentary hesitation, followed by a sudden accele. ration. The jerk is more severe after the joint has been undisturbed for some time, and especially on waking in the morning. After it has been repeated a number of times for experiment or observation it is painless and comparatively slight. It is never entirely absent. When the hand is quiet there is no pain except what is felt in chronic arthritis on pressure or when motion is forced beyond the point of comfort. Many of the uses of the hand are interfered with. A pencil cannot be sharpened in the usual way by flexion and extension of the fingers. It also impedes writing and sewing. A woman thus affected said that a needle in her fingers seemed as large as a pencil. Other manual movements are at times brought to a sudden stop in a surprising, inconvenient, and more or less painful way. The momentary pain is sometimes very severe.

Cases of this kind have been occasionally reported, and they have given rise to an extensive "literature" devoted largely to cause and mechanism. The peculiar action of a trigger finger resembles that seen when a pocket-knife is opened or shut. Movement is smooth till a square shoulder at the near end of the blade presents an obstacle which retards motion till a certain point is reached when motion is accelerated with a jerk. A counterpart of this obstacle may be found perhaps in a node on an articular surface where it would cause the halting action of a trigger finger. In the construction of a knife, however, an important feature is present which is absent from the anatomy of the hand, and that is the strong steel spring concealed in the handle of the knife. It produces positive pressure between the surfaces composing the metallic joint. If the spring were absent, or if its place were taken by a strip of some inert substance like cork, there would be no jerk, and shutting and opening the knife would be perfectly smooth. While there is nothing very closely resembling a steel spring in the hand or forearm, still we may find in muscular action a force which draws the surfaces together and creates a pressure comparable with that produced by the steel spring.

The fingers require not only delicacy and swiftness, but also strength. Small in bulk and weight, and far removed from the point of resistance in the inertia of the trunk, they still are endowed with great strength. Their power comes from important groups of muscular fibres assembled in the mass of the forearm. The long tendons of one set, the flexor sublimis, are inserted in the bases of the second phalanges. They make pressure on the articular surfaces, especially those of the nearest joint, the affected one in the case here reported, pressure augmented by the pull coming from the other group, the flexor profundus, whose tendons 
pierce those of the sublimis to be inserted in the distal phalanges. These tendons are bound to the phalanges by fibrous sheaths, and as they lie close to the bones and parallel with them a large part of their force is expended in pressing the articular surfaces together, the residue being given to moving the joint.

Motion is doubtless impeded by intra-articular irregularities in other parts of the body where joint surfaces are held in contact by the static resistance of circumarticular ligaments and to some degree by the dynamic influence of muscular contraction. No joints, however, but those of the digits are subject to the exceptionally great pressure of powerful muscles acting through tendons lying parallel to, and in close contact with, long bones. It is clear that when digital muscles are in action they concentrate on small articular surfaces pressure as positive and distinct as that made by the steel spring of the pocket knife.

After witnessing the dissection of a normal hand, by the kindness of Dr. Gallaudet, it appeared to me that the node present on the radial side of the base of the second phalanx of the middle finger of the affected hand might extend into the joint, where it would project as an obstacle on the palmar edge of the articular surface at the base of the phalanx. At this point it would be in apposition, when the joint is at a right angle, with the rounded lateral articulating condyle on the radial side of the head of the proximal phalanx. It is evident that when these two eminences, one abnormal and the other normal, pass each other, as they do when the joint traverses a right angle, they must, when pressed together by muscular contraction, experience a momentary hesitation followed by an acceleration of speed such as is seen in trigger finger, That there may be cases which do not have their origin in arthritis is suggested by the personal experience and research of my frinds, Dr. Weir and Dr. Abbe.

It is surmised that as the flexors are stronger than the extensors the jerk of trigger finger would be more severe in flexion than in extension. This is found to be the case. And further, it is found that the jerk is lessened or absent when passive is substituted for active motion. These and other experiments are interesting and sustain my proposition to a certain extent, but they are wanting in scientific accuracy and their results are not constant. Aside from these experimental observations the presence of unusual pressure between the joint surfaces when voluntary motion is made by the fingers and the probable presence of a node on one of the surfaces give sufficient warrant to the following proposition. In trigger finger an obstacle of arthritic origin on one of the articulating surfaces is surmounted with momentary hesitation followed by a jerk or sudden acceleration of speed, as the joint moves under the exceptional pressure which voluntary action interposes between the articular surfaces of the digital joints.

$X$ ray views have been taken in this case, but they fail to show any notable change in the bone. Ordinary inspection, however, shows swelling and deformity, with a projection on the radial side of the base of the second phalanx of the middle finger. The joint is an eighth of an inch wider at this point than its fellow of the other hand. Dissection would probably reveal surface irregularity. It is to be hoped, however, that a necropsy will not be proposed in the immediate future, as the joint herein described is part of the still good right hand of the author.
There has been no local treatment except by a gutter splint of sheet aluminium applied with a single strip of adhesive plaster to keep the joint extended at night. At the end of a year the jerk is less marked, but pain and stiffness continue and involve the metacarpo-phalangeal joint. Persistent catharsis has been maintained with oleum ricini, and as a result, perhaps, no other joint of the body has been attacked by the infection which is held to be the cause of chronic multiple arthritis, an affection intractable and as yet not well understood. New York.

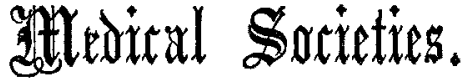

\section{ROYAL SOCIETY OF MEDICINE.}

\section{CLINICAL SECTION.}

Exhibition of Cases. - Spontaneous Gastro-enterostomy. A MEETING of this section was held on Dec. 11th, Sir Frederic Eve, the Vice-President, being in the chair

Dr. ALEXANDER MoRISON showed a case of Præcordial Thoracostomy for Heart Disease. The patient, a boy aged 16, was stated to have been in good health and capable of athletic exercise until Christmas, 1913. He had played football weekly from October till late in December. He then became breathless and had severe palpitation. No history of rheumatism or scarlet fever was obtainable. He was admitted to hospital in February, 1914, with tachycardia, irregular action of the heart, breathlessness, and a mitral systolic bruit. The area of cardiac dulness was much increased; powerful heaving of left ribs and cartilages from the fourth to the sixth and movement of the sternum were noticed. He was rendered comfortable by rest and taking tincture of digitalis, but without any diminution of thoracic movement, and was discharged in April feeling comfortable. He was told to return if again distressed, for præcordial thoracostomy. He did so in May and was again admitted. The operation was performed by $\mathrm{Mr}$. Mower White in June, when $4 \frac{1}{2}$ in. and $5 \frac{1}{2}$ in. of the fifth and sixth ribs and cartilages respectively were removed. No costopericardial adhesions were found. Since then he had been comfortable, but still required tincture of digitalis. The object of the operation was to relieve the enlarged heart of the labour of raising the chest wall as well as driving blood.

Dr. ARTHUR F. HerTz showed a case of Cardiac Achalasia (so-called cardiospasm). The woman, aged 36, had complained for ten years of pain under the lower part of the sternum and a choking sensation under the upper part whenever she ate. She had always had an impression that her food had difficulty in getting into her stomach. About two years ago she fell from a ladder and hit her chest; since then she had constantly vomited part of the food after every meal, and it came up quite undigested, but mixed with a considerable amount of mucous saliva. During the night she frequently woke owing to regurgitation of her evening meal through her nose; the food was now sour, especially if several hours had elapsed since the meal, in contrast to the food brought up during meals, which was never sour. The condition became steadily worse until nine weeks ago, when she ceased to be able to keep down any solid food at all. During the three days before she was seen six weeks ago, no food had been retained at all. An X ray examination showed that the whole osophagus was dilated, the obstruction being at the cardiac orifice of the stomach. There was violent peristalsis in the cesophagus, but the food only trickled with extreme slowness into the stomach. The condition was diagnosed as cardiac achalasia, and a mercury tube was passed. This met with no obstruction at the cardia, and the patient had since been able to retain her food, unless it was insufficiently masticated, by passing the tube immediately before each meal. The term "achalasia" ( $\alpha$, not; $\chi^{a \lambda a ́ c o, ~}$ I relax) was coined for him (Dr. Hertz) by Sir Oooper Perry to replace the term "spasm," which was incorrect for the following reasons: (1) Hypertrophy of the cardiac sphincter is never observed after death, even if the 